Case report

\title{
An unusual case of hypercapnic respiratory failure
}

\author{
Nimeh Najjar ${ }^{\mathrm{a}, *}$, Martin Cerda ${ }^{\mathrm{b}}$, Jorge Trabanco ${ }^{\mathrm{c}}$, Vinoo Ramsaran ${ }^{\mathrm{a}}$, James Cury ${ }^{\mathrm{a}}$ \\ a University of Florida College of Medicine, Pulmonary \& Critical Care, USA \\ ${ }^{\mathrm{b}}$ University of Florida College of Medicine, Cardiology, USA \\ ${ }^{\mathrm{c}}$ University of Florida College of Medicine, Internal Medicine, USA
}

\section{A B S T R A C T}

Asphyxiating thoracic dystrophy (ATD also known as Jeune syndrome) is a very rare disorder with an incidence in the United States of 1 case per 100,000-130,000 live births. Chronic alveolar hypoventilation leading to concurrent hypoxia is the main cause of morbidity and mortality in these patients due to its complications. A 22-year-old male with past medical history of ATD and severe kyphoscoliosis presented with progressively worsening shortness of breath for several days. Past surgical history was significant for multiple reconstructive sternal surgeries, his first surgery was at the age of two. His chest exam was without wheezing and was notable for symmetrically decreased breath sounds. Arterial blood gas showed PH 7.17, PCO2 155, PO2 95 and O2 saturation of $97 \%$ on $2 \mathrm{~L}$ nasal cannulae. PA and lateral chest xrays showed a long and severely narrow thoracic cavity. 2D echocardiography showed left ventricular ejection fraction of 55\% and evidence of severe pulmonary hypertension. CT chest angiography showed severe dilatation of the pulmonary artery in comparison to ascending aorta and significant right ventricular enlargement. Right heart catheterization confirmed these findings with a mean pulmonary arterial pressure of $61 \mathrm{mmHg}$. Within several days of hospitalization, patient developed acute worsening of his chronic hypercapnic respiratory failure thought to be due to worsening of pulmonary arterial hypertension with right heart failure. This in return was attributed to underlying ventilatory failure secondary to severe thoracic dystrophy. ATD is an autosomal recessive genetic disorder. Mutations in the IFT80 gene, which encode for an intraflagellar protein, cause this protein to be defective. Clinically, ATD is characterized by a small, narrow chest and variable limb shortness. While ATD is compatible with life, respiratory failure and infections are often fatal during infancy. Patients that survive past the age of 2 have seen respiratory complications resolve due to less pronounced thoracic malformations, but in our case the main cause of worsening pulmonary function was the degree of pulmonary arterial hypertension and right heart failure. Patients with ATD usually develop progressive hypercapnic respiratory failure due to an abnormally small thorax. Surgical options include lateral thoracic expansion or sternal and chest wall reconstruction. However, these surgeries only add a few years to survival without a definitive cure.

\section{Introduction}

Asphyxiating thoracic dystrophy (ATD also known as Jeune syndrome) is a very rare disorder with incidence in the United States of 1 case per 100,000-130,000 live births. Chronic CO2 retention in these patients is the main cause of morbidity and mortality due to its complications.

\subsection{Case narrative}

A 22-year-old Caucasian male with a past medical history of ATD and severe kyphoscoliosis presented with progressively worsening shortness of breath for several days. Past surgical history was significant for multiple reconstructive sternal surgeries, his first surgery was at the age of two. His chest exam was without wheezing and was notable for symmetrically decreased breath sounds. Arterial blood gas analysis showed PH 7.17, PCO2 155, PO2 95 and O2 saturation of 97\% on 2L nasal cannula. PA and lateral chest xrays showed a long and severely narrow thoracic cavity (Figs. 1 and 2). 2D echocardiography showed a left ventricular ejection fraction of 55\% and evidence of severe pulmonary hypertension. A computed tomography angiography (CTA) of his chest showed severe dilatation of the pulmonary artery in comparison to ascending aorta and significant right ventricular enlargement (Fig. 3). Right heart catheterization confirmed these findings with a mean pulmonary arterial pressure of $61 \mathrm{mmHg}$.

Within several days of hospitalization, the patient developed acute worsening of his chronic hypercapnic respiratory failure that was thought to be due to worsening of his pulmonary arterial hypertension with right heart failure. This in return was attributed to underlying ventilatory failure secondary to severe thoracic dystrophy.

\footnotetext{
* Corresponding author.

E-mail address: nimeh.najjar@jax.ufl.edu (N. Najjar).
} 


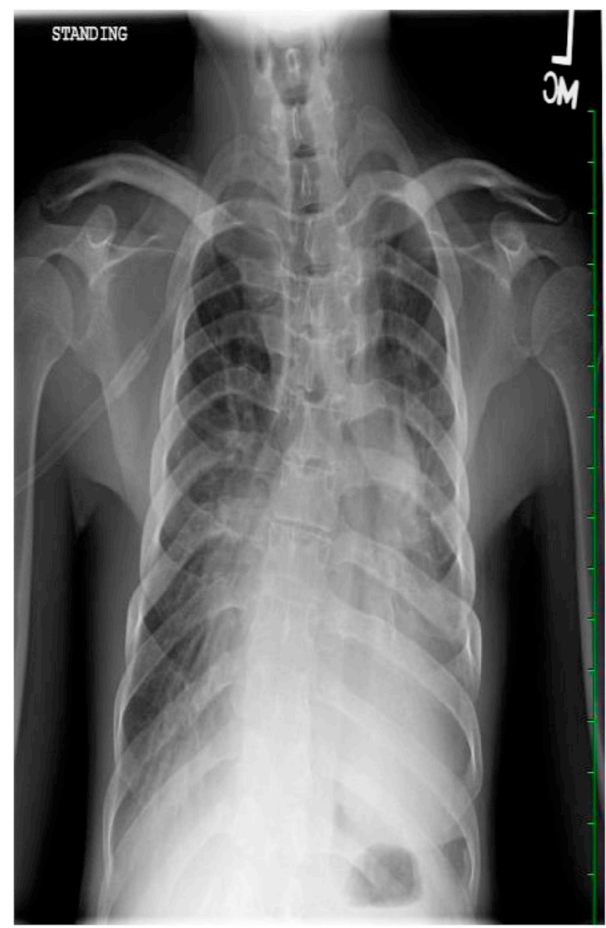

Fig. 1. PA chest xray showing an elongated and narrowed thoracic cavity.

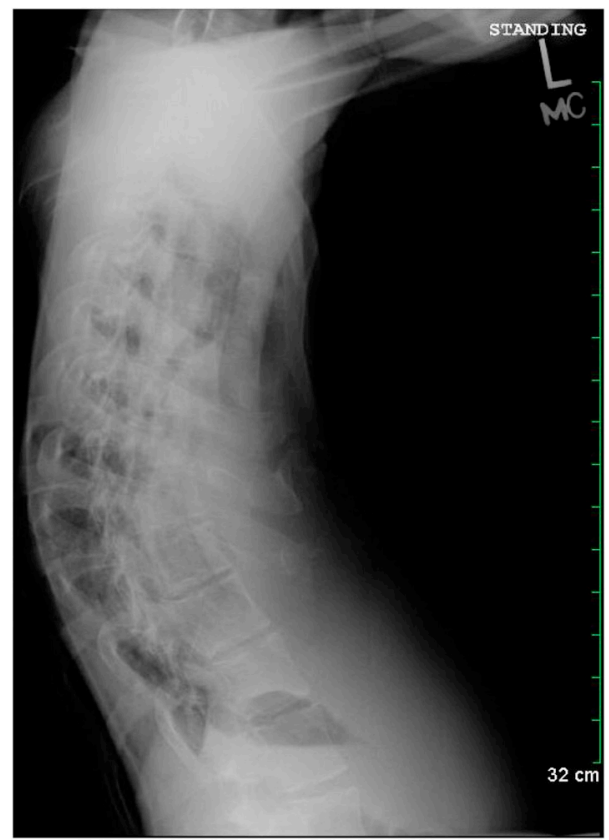

\section{Discussion}

ATD was first described by Jeune et al., in 1955 in two siblings with severely narrowed thoracic cavities [2]. Jeune syndrome is known to be genetically heterogeneous with an autosomal recessive mode of inheritance. Recently, found mutations in the IFT80 gene, which encode for an intraflagellar protein cause the protein to be defective, and are felt to lead to this syndrome. Clinically, ATD is characterized by a small, narrow chest and variable limb shortness [1]. The typical radiographic findings include a narrow, bell-shaped thorax with short, horizontally oriented ribs with irregular costochondral junctions, elevated clavicles, short iliac bones, hypoplastic phalanges of both hands and feet with cone-shaped epiphyses [3-5]. While Jeune syndrome is compatible with life, respiratory failure and infections are often fatal during infancy. In a recent article in the European Journal of Pediatrics, de Vries et al. described thirteen cases of Jeune syndrome ranging in age from 9 months to 22 years. Many complications are inherent in ATD; however, respiratory complications are of main concern during infancy [7]. Patients that survive past the age of 2 years usually see respiratory complications resolve due to less pronounced thoracic malformations, but in our patient the main cause of worsening pulmonary function was the degree of pulmonary hypertension and right heart failure. To our knowledge, this is the first patient reported with such severe pulmonary hypertension and right sided heart failure from ATD.

Although ATD and Jeune syndrome are often used interchangeably, the clinical manifestations are not limited to musculoskeletal abnormalities. Some authors emphasize the importance of recognizing this entity as a syndrome due to the effects this can have on cardiac, hepatic and renal function. This recognition becomes important especially in adults living with Jeune syndrome. Friedman et al. reported this syndrome in a 32 year old male with cardiac, renal and hepatic disease. Renal disease in ATD is thought to be both functional and anatomical $[6,7]$. The etiology of hepatic disease, however, is unclear. Friedman et al. postulated that prolonged neonatal jaundice, polycystic liver disease, bile duct hyperplasia and congenital hepatic cirrhosis may be thecause of hepatic disease in adulthood [8].

Patients with ATD usually develop progressive hypercapnic respiratory failure due to an abnormally small thorax requiring surgery. Chronic renal failure may require dialysis or transplantation. Liver disease is treated with phenobarbital or ursodeoxycholic acid [9]. Surgical options include lateral thoracic expansion or sternal and chest wall reconstruction. However, these surgeries only add a few years to survival without definitive cure. Our patient perhaps survived into early adulthood due to less severe renal and hepatic disease which likely reflects a different phenotype for the same underlying gene mutation known in Jeune syndrome. The chest wall reconstruction surgeries our patient had as a child may have added a few extra years to his life. However, the effects of chronic hypoventilation on cardiac function eventually led to his demise.

Fig. 2. Lateral chest xray showing an elongated and narrowed thoracic cavity.

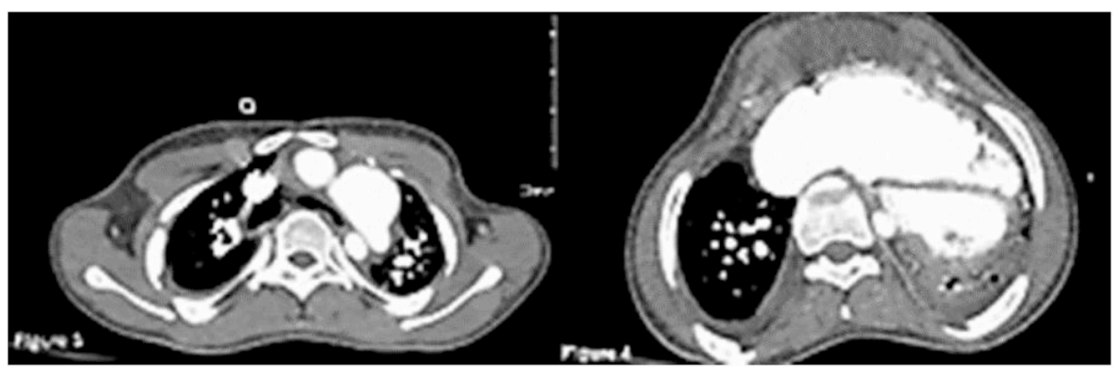

Fig. 3. Left shows a mediastinal view of CTA chest with a pulmonary artery that is larger than ascending aorta. Right shows mediastinal view of CTA chest with an enlarged right ventricle and a small left ventricular cavity. 


\section{Conflicts of interest}

\section{We have no conflict of interest to declare.}

\section{References}

[1] F. Oberklaid, D.M. Danks, V. Mayne, P. Campbell, Asphyxiating thoracic dysplasia: clinical, radiological, and pathological information on 10 patients, Arch. Dis. Child. 52 (1977) 758-765.

[2] M. Jeune, C. Beraud, R. Carron, Dystrophie Thoracique asphyxiante de caractère familial, Arch. Fr. Pediatr. 12 (1955) 886.

[3] R.C.M. Hennekam, F.M. Beemer, L.J. Gerards, B. Cats, Thoracic pelvic phalange dystrophy (Jeune syndrome), Tijdschr. Kindergeneeskd. 51 (1983) 95-100.
[4] R.C. Herdman, L.O. Langer, The thoracic asphyxiant dystrophy and renal disease, Am. J. Dis. Child, 116 (1968) 192-201.

[5] T. Pirnar, E.B.D. Neuhauser, Asphyxiating thoracic dystrophy of the newborn, Am. J. Roentgenol. 98 (1966) 358-364.

[6] J. de Vries, J.L. Yntema, C.E. van Die, N. Crama, E.A.M. Cornelissen, B.C.J. Hamel, Jeune syndrome: description of 13 cases and a proposal for follow-up protocol, Eur. J. Pediatr. 169 (2010) 77-88.

[7] A.B. Gruskin, H.J. Baluarte, M.L. Corte, I.B. Elfenbein, The renal disease of thoracic asphyxiating dystrophy, Eur. PMC 10 (4) (1974) 44-50.

[8] J.M. Friedman, H. Kaplan, J. Hall, The Jeune syndrome (asphyxiating thoracic dystrophy) in an adult, Am. J. Med. 59 (6) (1975) 857-862.

[9] M.B. O'Connor, D.P. Gallagher, E. Mulloy, Jeune syndrome, Postgrad. Med. 84 (2008) 559. 